\title{
30 : 2: A Game Designed to Promote the Cardiopulmonary Resuscitation Protocol
}

\author{
Imma Boada, ${ }^{1}$ Antonio Rodriguez-Benitez, ${ }^{1}$ Juan Manuel Garcia-Gonzalez, \\ Santiago Thió-Henestrosa, ${ }^{2}$ and Mateu Sbert ${ }^{1,3}$ \\ ${ }^{1}$ Graphics and Imaging Laboratory, University of Girona, 17071 Girona, Spain \\ ${ }^{2}$ Departament Informatica, Matemàtica Aplicada i Estadística, University of Girona, 17071 Girona, Spain \\ ${ }^{3}$ School of Computer Science and Technology, Tianjin University, Tianjin 300072, China
}

Correspondence should be addressed to Imma Boada; imma.boada@udg.edu

Received 8 January 2016; Accepted 11 April 2016

Academic Editor: Michela Mortara

Copyright (C) 2016 Imma Boada et al. This is an open access article distributed under the Creative Commons Attribution License, which permits unrestricted use, distribution, and reproduction in any medium, provided the original work is properly cited.

Cardiopulmonary resuscitation (CPR) is a first-aid key survival technique used to stimulate breathing and keep blood flowing to the heart. Its effective administration can significantly increase the survival chances of cardiac arrest victims. We propose $30: 2$, a videogame designed to introduce the main steps of the CPR protocol. It is not intended for certification and training purpose. Driven by the 2010 European Resuscitation Council guidelines we have designed a game composed of eight mini games corresponding to the main steps of the protocol. The player acts as a helper and has to solve a different challenge. We present a detailed description of the game creation process presenting the requirements, the design decisions, and the implementation details. In addition, we present some first impressions of our testing users ( 25 children, five of each age from 8 to 12 years old and 12 males and 13 females). We evaluated clarity of instructions and three settings of the game: the aesthetics of scenarios, the playability, and the enjoyability of each mini game. All games were well punctuated, and there are no significantly differences between their sex. The proposed game can be a suitable tool to disseminate and promote CPR knowledge.

\section{Introduction}

In the last years, computer-based learning has emerged as an effective tool to promote learning and develop cognitive skills [1]. This approach combines serious learning with interactive entertainment providing many benefits in terms of learning and motivation [2-5]. According to Kebritchi and Hirumi [6], there are five main reasons for the effectiveness of computer-based learning: first, it uses action instead of explanation; second, it creates personal motivation and satisfaction; third, it accommodates multiple learning styles and skills; fourth, it reinforces mastery of skills; and fifth, it provides an interactive and decision-making context. In addition, computer games provide other benefits such as complex and diverse approaches to learning processes and outcomes, interactivity, ability to address cognitive as well as affective learning issues, and motivation for learning [7].
If we consider all these benefits and also that there is an extended use of portable gaming platforms among young people, we can conclude that computer-based games are a perfect channel to promote learning contents $[8,9]$. In this context, we propose $30: 2$, a computer game designed to promote the cardiopulmonary resuscitation protocol among citizens and especially among children. The game is not intended for certification and training purpose but to increase awareness and improve knowledge about cardiopulmonary resuscitation.

Cardiopulmonary resuscitation (CPR) is an emergency lifesaving procedure that is done when someone's breathing or heartbeat has stopped. The procedure combines rescue breathing, to provide oxygen to the lungs, and chest compressions to keep oxygen-rich blood flowing until the heartbeat and breathing can be restored. Permanent brain damage or death can occur within minutes if blood flow stops. However, 
immediate CPR can double or triple survival rates and, CPR plus defibrillation within 3 to 5 minutes of collapse, can produce survival rates as high as 49 to $75 \%$ [10-14].

Since 1960, when Kouwenhoven et al. [15] published an article stating that anyone, anywhere, could perform CPR, providing CPR has become an essential competency not only for expert but also for lay people. Different organizations, such as the European Resuscitation Council (ERC) [14], the Red Cross, and the American Heart Association [16], have defined guidelines that describe how resuscitation should be undertaken both safely and effectively. In addition, many countries have initiated CPR programmes to train lay rescuers in CPR and several strategies have been used to teach it including mass training [17], or video self-instruction [18], among others. Unfortunately, the rate of bystander CPR at cardiac arrest is still very low, less than 20\% [19]. There is a lack of awareness of the availability of CPR training for the public and lack of interest [20]. Therefore, any proposal that enhance CPR dissemination and promotion will be welcome. With this idea in mind, we created $30: 2$ a computer game designed to introduce, in an enjoyable way, the main steps of the CPR protocol to children and lay people. The game is composed of eight mini games corresponding to the main steps of the CPR protocol proposed by the ERC 2010 [14]. Each mini game presents a different challenge and different levels of difficulty to maintain the attention of the player. The game name, $30: 2$, comes from the key step of the CPR protocol that performs 30 compressions and 2 ventilations.

\section{Related Work}

In this section, we briefly describe the CPR protocol which is the basis of our game. Then, we review different applications that have been proposed to learn this protocol and some of the studies that have been carried out to promote it among nonexpert audience.

2.1. The Basic Life Support Protocol. The basic life support protocol defines the procedures that have to be carried out when a victim of a cardiac arrest is found. We will follow the protocol defined by the ERC 2010 [14] and represented in Figure 1. According to this protocol we have to proceed as follows. (1) Check the perimeter to make sure the situation is safe. (2) Check the victim for a response by gently shaking his shoulders and asking loudly if he is all right. (3a) If the victim responds, leave him in the position in which you found him ensuring there is no further danger; try to find out what is wrong with him/her and get help if needed. (3b) If he victim does not respond, shout for help, turn the victim onto his back, and then, open the airway placing your hand on his forehead and gently tilt his head back with your fingertips under the point of the victim's chin; lift the chin to open the airway. (4) Keeping the airway open, look, listen, and feel for breathing. (5a) If he is breathing normally, turn him into the recovery position. Send or go for help (call 112 or local emergency number for an ambulance) and continue to assess that breathing remains normal. (5b) If the breathing is not normal or absent, send someone for help and to

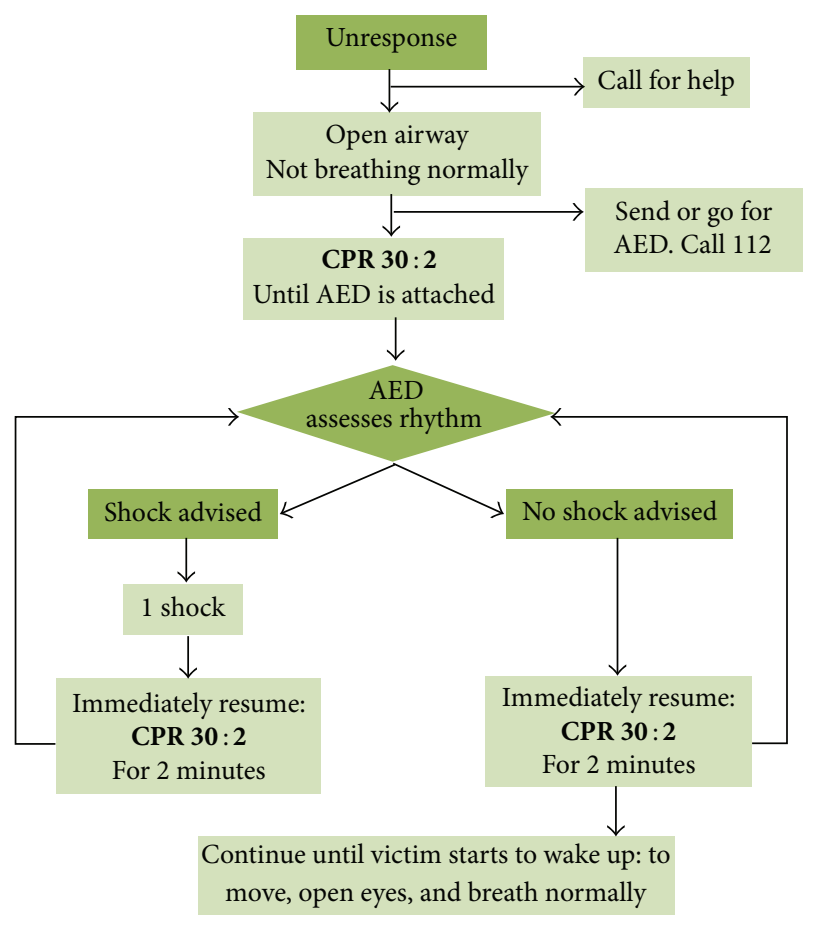

FIgURE 1: Automated external defibrillator algorithm according to 2010 ERC guidelines.

find and bring an automatic external defibrillator (AED) if available; or if you are on your own, use your mobile phone to alert the ambulance service. Leave the victim only when there is no other option. (6) Start chest compression and rescue breaths in a ratio of $30: 2$. To perform compressions, you must place your hands vertically above the victim's chest and press down on the sternum. Pressure depth has to be between 5 and $6 \mathrm{~cm}$ and at a rate of at least 100 compressions per minute, but not exceeding 120. After 30 compressions open the airway again and perform two rescue breaths, blowing steadily into the mouth while watching for the chest to rise, taking about 1 second as in normal breathing. Continue with chest compressions and rescue breathes, in a ratio of $30: 2$, and stop to recheck the victim only if he starts to wake up: to move, open eyes, and breathe normally. Otherwise, do not interrupt resuscitation. (7) If the victim recovers, put him in the lateral security position.

2.2. State of the Art on CPR Applications. Different methods have been proposed to teach CPR. Below we describe some of them (see Table 1 for a summary).

On the one hand, there are video training applications such as Save-a-Life simulator [21], an interactive online video simulation that tests the player knowledge of helping someone suffering from sudden cardiac arrest, and CPR-andChoking [22], an application that provides instant information on how to perform CPR and how to aid a choking victim. On the other hand, there are handheld device applications such as CPR Game [23], a cardiac arrest simulator on iOS platform focused on advanced CPR training; iResus [24], an application for smart phone, designed to improve 
TABLE 1: Some of the proposed applications to introduce CPR or some of the steps of the protocol.

\begin{tabular}{|c|c|}
\hline $\begin{array}{l}\text { Application } \\
\text { type }\end{array}$ & \\
\hline $\begin{array}{l}\text { Video } \\
\text { training }\end{array}$ & Save-a-Life simulator [21], CPR-and-Choking [22] \\
\hline $\begin{array}{l}\text { Handheld } \\
\text { device }\end{array}$ & $\begin{array}{c}\text { CPR Game [23], iResus [24], iCPR [25, 26], } \\
\text { M-AID [27], CPR simulator [21], and LifeSaver } \\
\text { [28] }\end{array}$ \\
\hline PC platform & Mini-VREM [29], AED Challenge [30] \\
\hline $\begin{array}{l}\text { Serious } \\
\text { games }\end{array}$ & $\begin{array}{l}\text { JUST [31], MicroSim [32], Staying Alive [33], } \\
\text { LISSA [34], Relive [35], Viva!Game [36], and } \\
\text { HeartRun [37] }\end{array}$ \\
\hline
\end{tabular}

the performance of an advanced life support provider in a simulated emergency situation; $\operatorname{iCPR}[25,26]$, an iPhone application designed for both lay persons and healthcare professionals able to detect the rate of chest compressions performance by using the built-in accelerometer; M-AID [27], a first-aid application for mobile phones that uses yes or no questions to judge an ongoing situation giving to the user detailed instructions of how to proceed; CPR simulator [21], a set of CPR exercises including adult, child, and infant CPR simulator that runs through the CPR sequence; and LifeSaver [28] a simulator proposed by the UK Resuscitation Council that fuses interactivity with live-action film. In addition, some applications for PC platforms are Mini-Virtual Reality Enhanced Mannequin (Mini-VREM) [29] which is a CPR feedback device with motion detection technology including Kinect, sensor and software specifically designed to analyse chest compression performance and provide realtime feedback in a simulation training setting; and AED Challenge [30], an application that provides online automated external defibrillation and CPR skill practice and testing with realistic scenarios. Finally, in the serious games context, some games for CPR training are JUST [31], an immersive virtual reality situation training system for nonprofessional health emergency operators; MicroSim Prehospital [32] designed for prehospital training on emergency medical services; and Staying Alive [33], an online 3D simulator which provides a learning experience of saving a virtual patient from cardiac arrest in four minutes. LISSA [34] is a serious game with actions turning around an emergency situation represented in a $3 \mathrm{D}$ virtual environment with the victim, the helper, and all the auxiliary tools that may require the emergency. The player has to save the victim applying the CPR actions that are evaluated and used to update the game score. When game is over LISSA returns a performance report. This game is used to complement CPR teaching. Other serious games are Relive [35], a first person 3D adventure where the player faces different rescue situations and can test the quality of his CPR directly; Viva!Game [36], a Web-based serious game designed to create awareness on cardiac arrest and cardiopulmonary resuscitation; and HeartRun [37], a mobile simulation game to train resuscitation and targeted at giving school children an understanding of CPR and getting them to take action.
Focusing on the audience, different experiences have been carried out to introduce CPR among children. Uray et al. evaluated the feasibility of life supporting first-aid training as a mandatory subject for 6-7-year-old children of primary schools [38]. They stated that resuscitation skills should be learnt at school, since children are easily motivated, learn quickly, and retain skills [39]. Jones et al. indicated that children of 9 years should be taught to perform CPR, including chest compressions [40]. In addition, the good results obtained in different experiences that have been carried out all over the world have motivated associations such as the ERC, the American Heart Association, and the American Academy of Pediatrics to recommend teaching resuscitation to school children [41-43].

From previous experiences, it makes sense to consider the development of a new game to introduce the CPR protocol to children. In addition, considering the current state of the art, we have seen that most of reported methods are based on multimedia approaches where video, text, and audio are combined to reproduce in a practical case the CPR protocol. In some cases, users can also interact by answering questions that drive them to the correct sequence of the protocol. A similar approach is followed in the case of serious games where instead of real actors they use avatars. Finally other applications just focus on the CPR maneuver. In our case, we propose a different approach to engage children attention on CPR. The axis of our game is also the CPR protocol but we consider the protocol steps independently. For each step, we create a mini game with a challenge that helps the user to retain the key procedure of the step, with enough variety of challenges to maintain the attention of the player and avoid boring him doing the same action for a long time.

\section{Game Description}

To describe the $30: 2$ videogame, we are going to follow the educational game development approach (EGDA) proposed by Torrente et al. [44]. This approach covers all tasks from game design to implementation and evaluation. It is built around four basic principles. The first is a procedure-centric approach that gives importance to capturing the procedural knowledge of the domain. In our case, this knowledge is provided by the ERC protocol that determines the steps that have to be carried out in case of sudden heart attack (see Section 2.1). The second is the collaboration between experts. In our case, the development team works in close collaboration with physicians specialized in cardiopulmonary resuscitation. The third is the agile development with agile tools. In our case, we apply an iterative process that includes analysis, game design, implementation, and quality assurance. The fourth is the low-cost game model. In our case, due to budget restriction our proposal considers $2 \mathrm{D}$ animations instead of $3 \mathrm{D}$ realistic models that require a much bigger budget. Below, we give a more detailed description of the main steps of the development process.

3.1. Analysis. The analysis phase was done in collaboration with experts on CPR that are in charge of CPR instruction 

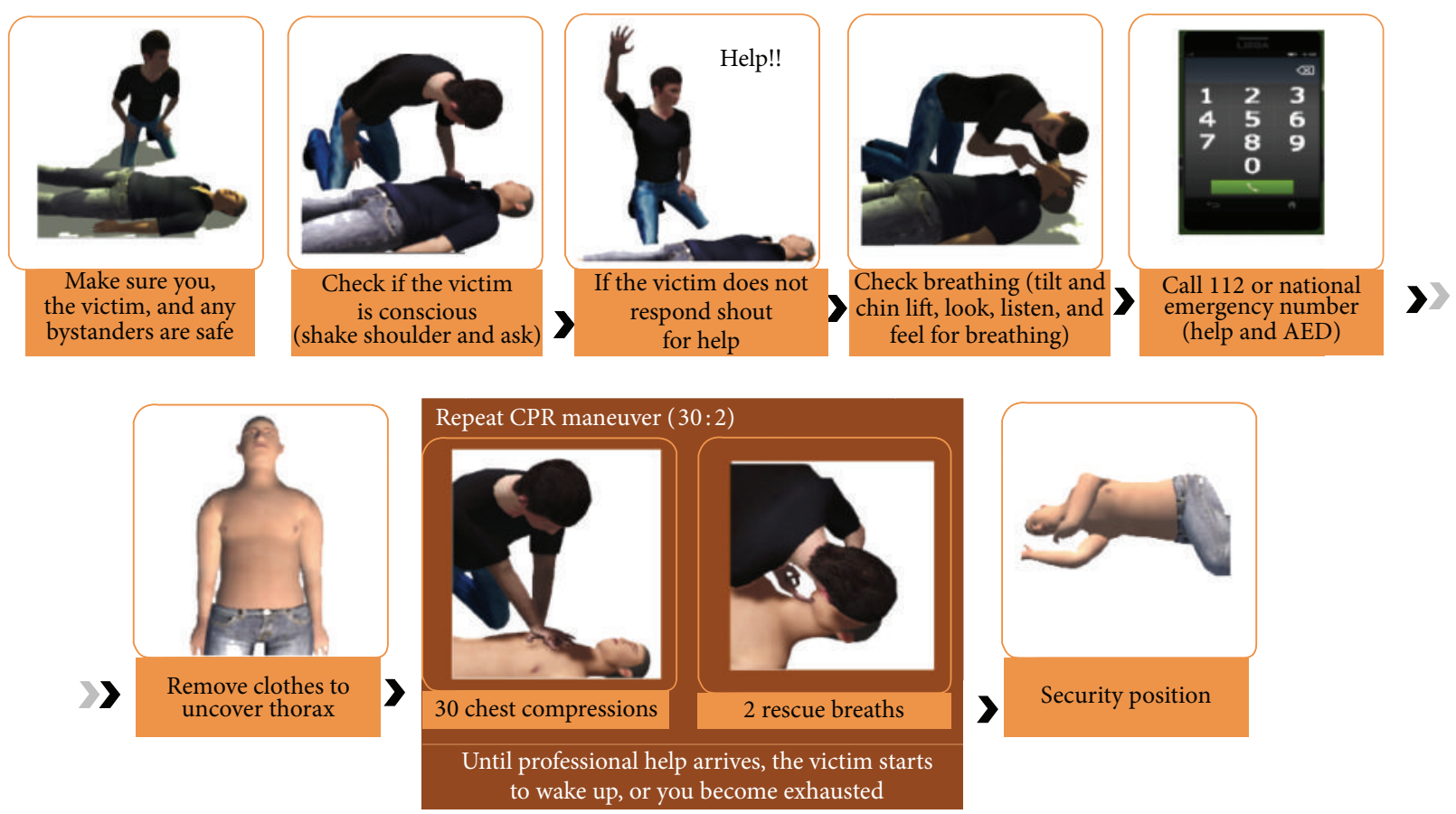

FIGURE 2: The steps of the basic life support protocol that have been selected to define the proposed game. We will create one mini game per selected step.

at our university and also at middle schools. For the school courses, they present the ERC guidelines to children, using the material provided by the ERC, and they also use a mannequin to practice. The purpose of the courses is to make children familiar with CPR concepts. The $30: 2$ game was conceived as a complement to these classes and as a way to preserve the acquired CPR knowledge. With this idea in mind, to delineate the objectives of our game, we considered the ERC guidelines presented in Section 2.1 and, assessed by the experts, we identified the steps and the key procedures of the protocol to be considered. The selected steps are represented in Figure 2 and correspond to the main case of the protocol in which the victim requires the CPR maneuver. We do not take into account the situations described in step (3a) or (5a).

The pedagogical approach used to design the game is based on the experiential learning theory, where educators aim engaging learners in direct experience to increase their knowledge, skills, and values [45]. Experience occurs as a result of interaction between human beings and an environment in forms of thinking, seeing, feeling, handling, and doing [45]. In our case, this experience is going to take place in an artificial environment where a victim has to be recovered from a heart attack by applying the CPR protocol. There are five instructional strategies rooted in the concept of learning through experiences. These are learning by doing, experiential, guided experiential, case-method teaching, and combination of experiential and inquiry-based learning [6]. In our case, we considered the learning by doing strategy. Schank et al. [46] posited seven instructional events to facilitate this experience. In the analysis phase, we defined these instructional events as follows:

(1) Goals definition: our objective is to introduce the main steps of the CPR protocol to the children. With this purpose we decided to create eight mini games, one for each CPR step.

(2) Mission: the mission is to save a heart attack victim by correctly applying the CPR steps. This mission is decomposed in eight submissions one for each CPR step.

(3) Cover story: there is a heart attack victim in a park and the player has to save him. We decided to create a common scenario for all the mini games and we adapted it to each mini game's requirements.

(4) Roles: the player who acts as a rescuer.

(5) Operation scenarios: all mini games take place in a park; we will provide different views of the park depending on the actions to be carried out.

(6) Provided resources: each mini game has a different challenge. Provided resources will be adapted to it. Game instructions will be given using the keywords of the protocol steps.

(7) Provided feedback: when the challenge is achieved a well done message will appear as well as the keywords of the protocol step.

In addition, we will support different levels of difficulty to keep a high level of engagement. We will support two playing 
modes. In the first mode, the player will play mini games individually, one by one to reach the maximum level of each mini game. In the second mode, the player will follow the complete sequence of mini games at the same level to see the whole protocol each time. For the CPR courses, we consider that the second mode is the most suitable. To increase the chance to perform the whole CPR process, we give five lives to the player, represented as hearts. In this way if the player fails in one step he has the chance to repeat it. Having several lives, we consider that players engagement in the game will increase as well as, in turn, their intrinsic motivation [47, 48]. In addition, we also use written positive feedback to increase feelings of competence and autonomy, which in turn lead to increased motivation [49].

3.2. Game Design. To design the game, we considered that the player acquires the role of a helper who has to save the victim of a cardiac arrest by applying the main steps of the CPR protocol. To guide the player between mini games at the beginning of each one we present the instructions of the step protocol as highlighted keywords, with the goal that the player could retain them easily. In addition, at the end of the mini game, we show the obtained score and the achieved steps. The player has five lives represented as five hearts. To solve the mini game challenge we have a predefined time that is defined according to the level of difficulty. Below we describe the different mini games. For each mini game, we will describe its pedagogical objective which is related with some procedure of the protocol, the scenario, the challenge, the interaction required to achieve the challenge, how we create the different levels of difficulty, and the messages with keywords.

3.2.1. Mini Game 1 (Isolate Victim). This mini game is related with step (1) of the protocol. Its pedagogical objective is to transmit to the player that when there is a victim the helper has to check the perimeter to make sure the situation is safe. We designed a game where the main scene is a park with a lot of people. People are represented as hearts of different colours and the victim as a broken heart. All hearts are on movement and the player has to press on the broken heart. Then, it will appear a security perimeter represented as a circle around the victim. The player has to move hearts out from the perimeter to isolate the victim. In this case, the player interacts by pressing on the broken heart and dragging and dropping on the hearts inside the security position. To create the different levels of difficulty we increase the number of hearts and also the used colours. At the beginning, all hearts have the same colour and then, as level of difficulty increases, new colours are added. In addition, time will be adapted to the level, allotted time decreases when difficulty level increases.

The text that appears in the game corresponds to keywords of the represented step. Before starting the game, a screen appears with the current score, the level of difficulty, and the game instructions. In this case, the text is find and isolate the victim. Once the game starts, the message find the victim and isolate it appears. At the end, the message victim is in a secure position appears.

3.2.2. Mini Game 2 (Check Conscious). This mini game is related with step (2) of the protocol. Its pedagogical objective is to transmit that to check the victim conscious; the helper has to shake his shoulder and ask if he is all right. We designed a game where the scene is a frontal view of the victim with the helper hand on the victim shoulder. In addition, there are glyphs with a question mark on the screen. These are of different colours and shapes; some icons add points and others subtract. Each time we click on a correct glyph, the hand will move victim shoulder to simulate the shaking procedure. The player has to achieve as much points as possible by pressing the correct glyphs. In this case, the player interacts by pressing on the correct icons. The levels of difficulty are related with the number of glyphs that appear and also their speed; the greater the difficulty, the greater the number of glyphs and speed. In addition, playing time will be adapted to the level; allotted time decreases when difficulty level increases.

As in the previous mini game the text that appears in the game corresponds to keywords of the represented step. Before starting the game, a screen appears with the current score, the level of difficulty, and the game instructions which are check victim's response. Once the game starts the message press blue and yellow glyphs appears. In the middle of the game the message are you all right appears and at the end, the message victim is unconscious appears.

3.2.3. Mini Game 3 (Ask for Help). This mini game is related with the first part of step ( $3 \mathrm{~b}$ ) of the protocol. Its pedagogical objective is to transmit that if the victim is not conscious, the helper has to shout for help. We designed a game where the scene represents a helper beside the victim. The helper has a speech balloon that has to be completed with the word help. Glyphs with letters fall down from the top of the screen and the player has to select the letters to write help. If letters are not selected in the correct order, he has to start again. The player has to complete the help word in the available time. In this case, the player interacts by pressing on the glyphs with the correct letters. The levels of difficulty are related with the falling speed of icon letters. In addition, time will be adapted to the level; allotted time decreases when difficulty level increases.

Again the text that appears in the game corresponds to keywords of the represented step. Before starting the game, a screen appears with the current score, the level of difficulty, and the game instructions which are Ask for Help. When the game starts the text press the correct letters appears as well as, at the end, well done! Help is on the way!

3.2.4. Mini Game 4 (Check Breath). This mini game is related with the first part of step (3b) and step (4) of the protocol. Its pedagogical objective is to transmit that if the victim is not conscious, in order to check breath the helper has to perform the tilt and chin lift by correctly placing hands on the head and move the victim's head back to open air way. Then, look 
at the thorax to see if it is moving and, finally, listen if the victim is breathing. We designed a game with the victim lying on the floor. We have a lateral view of his body and different glyphs representing right and left hands, ears, and eyes that will appear on the screen. The player has to select the hands and drop them to the correct head position to perform the tilt and chin lift. Then, place an eye glyph on the thorax and the ear near the victim's face. In this case, the player interacts by pressing on the glyph and dragging it to the correct position. The levels of difficulty are related with the number of glyphs and their speed. In addition, time will be adapted to the level; allotted time decreases when difficulty level increases.

The message that appears before starting the game is check breath by performing tilt-chin maneuver; look at the thorax and listen respiration. Once the game starts the messages drag hands to correct positions and place eye on the thorax and ear on the face appear. The final message is victim is not breathing.

3.2.5. Mini Game 5 (Call Emergency). This mini game is related with step (5b) of the protocol. Its pedagogical objective is to transmit that if the victim is not breathing, the helper has to call emergency number and ask for a defibrillator. We designed a game where the scene represents the front face of a mobile showing the numbers and the screen to see the dialed numbers. The player has to dial the correct emergency number in the available time. In this case, the player interacts by pressing on the phone numbers. To obtain different levels of difficulty the phone numbers change their position. In addition, time will be adapted to the level; allotted time decreases when difficulty level increases.

The message that appears before starting the game is call emergency and ask for a defibrillator. Once the game starts the message call emergency appears as well as, at the end, well done! Emergency is on the way.

3.2.6. Mini Game 6 (Remove Clothes). This mini game is related with step (6) of the protocol. Its pedagogical objective is to transmit that we need to uncover the thorax of the victim to perform the CPR maneuver. We designed a game where the scene represents a front plane of the victim with different layers of clothes (a scarf, a jacket, a shirt, etc.). The player has to remove, in the available time, all the clothes until thorax is uncovered. In this case, the player interacts by pressing on the screen following the shape of the scarf and the zip or pressing on the buttons. The levels of difficulty are related with the layers of clothes, which increase with the level of difficulty. In addition, allotted time will be adapted to the level; allotted time decreases when difficulty level increases.

The message that appears at the beginning is remove clothes to uncover thorax. Once the game starts, the message remove clothes appropriately appears as well as, at the end, well done! Thorax uncovered.

3.2.7. Mini Game 7 (Perform CPR). This mini game is related with step (6) of the protocol. Its pedagogical objective is to show the key maneuver of the CPR protocol. The player should learn how to place hands position and where, the rhythm, and the number of compressions that has to be done. In addition, he has to learn that after compressions he has to perform two rescue breaths in a ratio of $30: 2$. Moreover, he has to learn that compressions are at least of $5 \mathrm{~cm}$. We designed a game with a set of icons to select correct hand positions and placement. In addition, there is a central menu with two icons one for compressions and the other for ventilations, an indicator to show compression depth and another to indicate ventilation degree. This menu has a central board to indicate if maneuver is correct or not. The four considered degrees of correctness are critic, bad, good, and perfect. The player has to perform compressions and ventilations correctly. The player interacts pressing on the correct icons. In this case, the levels of difficulty are related with the accuracy at which CPR maneuver is performed.

The message that appears at the beginning is perform $C P R$ maneuver. Once the game starts, the messages that appear are select correct hands position, perform $30: 2$, and put defibrillator patches. At the end, the final message is well done! The victim has recovered.

3.2.8. Mini Game 8 (Security Position). This mini game is related with the last step of the CPR protocol. Its pedagogical objective is to show the movements that have to be done to place the victim in the lateral security position. In this position, the mouth is downward so that fluid can drain from the patient's airway; the chin is well up to keep the epiglottis opened. Arms and legs are locked to stabilize the position of the patient. We designed a game where the scene represents a lateral view of the victim. The player has to press on the correct parts of the body and perform the correct movements to place the victim in the security position. The player interacts by pressing on the correct part of the victim's body and dragging it to the correct position. In this case, the levels of difficult are related with the available time which decreases when difficulty level increases.

The message that appears at the beginning is place the victim in the lateral security position. Once the game starts the message that appears is press on the body parts and move and at the end well done! Victim is in lateral security position.

3.3. Implementation. To implement the game we used Unity3D, a cross-platform game engine, that can support Mac OSX and WindowsXP/Vista/7/8. It supports three scripting languages: JavaScript, C\#, and a dialect of Python called Boo. All three are equally fast and interoperate, and can use the underlying. NET libraries support databases, regular expressions, XML, file access, and networking. Although scripting is frequently considered as limited and slow, in Unity3D scripts are compiled to native code and run nearly as fast as $\mathrm{C}++$. In the project, all the game scripts have been programmed in C\#.

The proposed game has been programmed as a finite state machine, where each state can contain different state machines. We used a generic home-implemented state machine library to implement the game flow. This library allows the definition of each state machine, the states that compose it, the actions each state will perform, and its transitions, in a very detailed way. The library only needs 


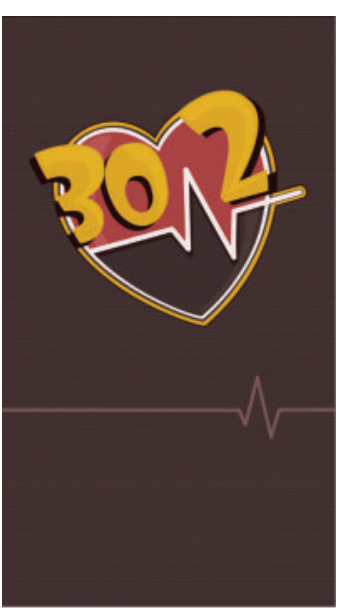

(a)

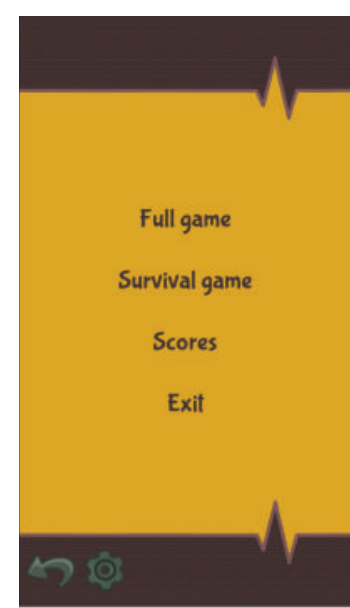

(b)

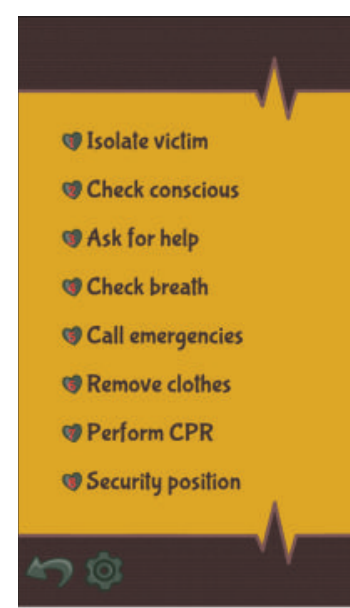

(c)

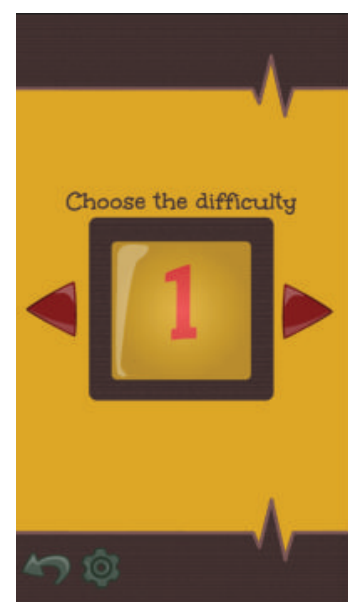

(d)

FIGURE 3: Game screens corresponding to (a) the main page and the menus to select (b) the playing mode, (c) the mini game, and (d) the difficulty level.

the user to define what a particular element requires: (i) for each state machine, we have to define the first state to be executed; (ii) for each action, we define the actual action that it will perform; (iii) for each transition, we define the condition for the transition and the next state to which it will move if condition is true (this allows the reutilization of transitions if the condition and destination match); and (iv) in each state, we define the list of actions that it will perform, the substate machines it contains (if any), the transitions it has, and if it is a final state. All the implementation references are generic and are checked during compilation. In this way, we ensure that all references are correct and avoid the continuous errors search. In addition, since elements are not instantiated until they are needed, performance increases considerably. The library also contains a static class that is used as a log. This class allows the subscription of delegates to the class, so that subscribed functions receive the log messages. We have also implemented a library to control scores. This library stores the score independently for each level, allowing us to personalize each level and how it calculates its score and stores them in a manager that allows us to determine what to do with the obtained scores. An important feature of the proposed implementation is that it can be easily upgradable to new CPR guidelines.

When the user starts the game, the general state machine will be initialized and this will initialize player information such as the score. The first state of this machine is the first mini game, Isolate Victim. When the Isolate Victim mini game starts, the difficulty level information and the submachine of the selected level are initialized. In the first state of this submachine, the user has to press on the broken heart. The action that allows pressing on all the hearts will be activated. When the player presses a heart it checks if it is the broken one or not. If it is an unbroken one, it will remain in the same state; otherwise, it will activate the transition to go to the next state. In the new state, the action to remove unbroken hearts from the perimeter of the broken one will be activated.
In this state, we continuously control that no hearts are into the perimeter. If there are, it remains in the same state. Once no more hearts are into the perimeter no more transitions are possible. Since this state is the last one, the submachine will finish and will inform its father state that it has finished. The father state will notify the score of the completed level to be stored in the score manager. Then, according to the game mode and the level, it will go to the next mini game or to the next level of the same mini game.

3.4. Quality Assurance. To ensure the game was stable and free of programming errors we continuously perform tests at different levels. We also used as testers members of our laboratory not related with the project but familiar with video games. Once all mini games were approved by our team, we performed tests with nonexperimented users with special attention to children. During tests we analysed playful experience to ensure that mini games were enjoyable enough. During these tests different proposals were done by the users and these led to different modifications on the mini games. To test the educational value after a testing session, we asked users to write the eight steps of the protocol to evaluate if they were able to reproduce them. In all the tests, they were able to do it.

\section{Results}

To present the results we will show different screens of the game grouped by mini games. For a complete demo of the game you can visit http://gilabparc.udg.edu/jocs/30_2/trailer .mov.

First images corresponding to some of the menus are presented in Figure 3. From (a) to (d), we can see the main screen with the game logo (see Figure 3(a)); the menu to select the playing modes (Full to play all the steps of the protocol, Survival to achieve the upper level of one mini game before 


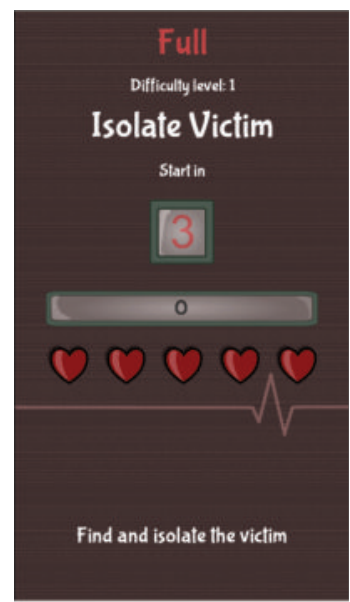

(a)

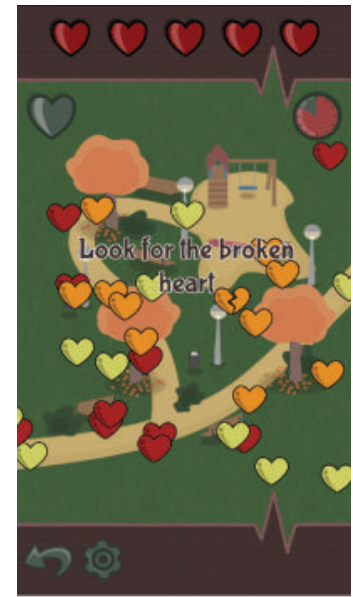

(b)

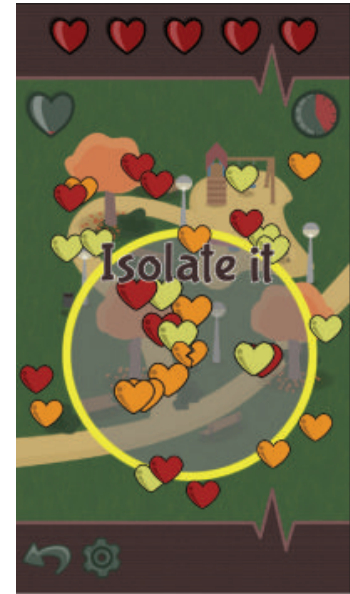

(c)

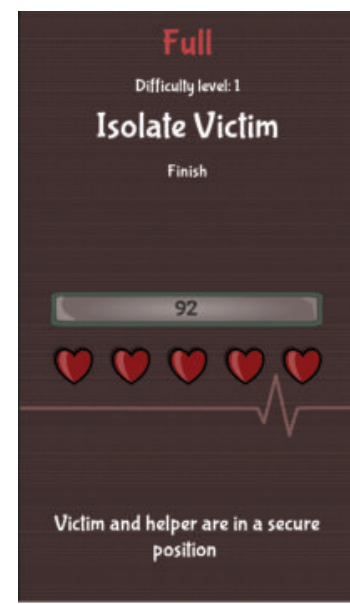

(d)

FIGURE 4: Screens of the Isolate Victim mini game. (a) Init screen with game instructions. (b) Before detecting the broken heart. (c) After detection of victim with the security perimeter from which hearts have to be removed. (d) Final screen where we can see the game score and the message that victim and helper are in a secure position.

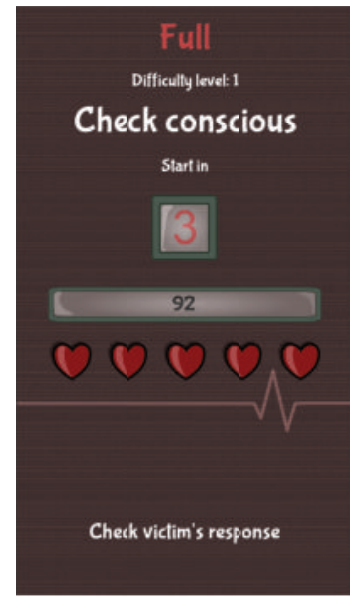

(a)

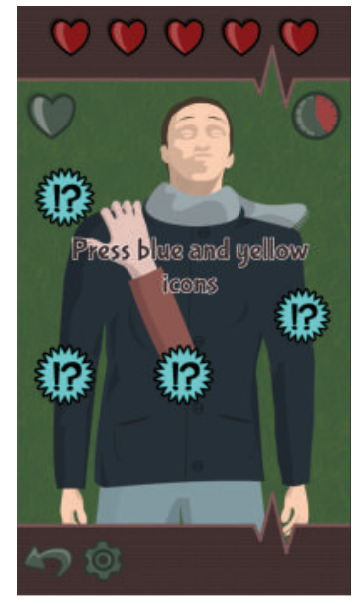

(b)

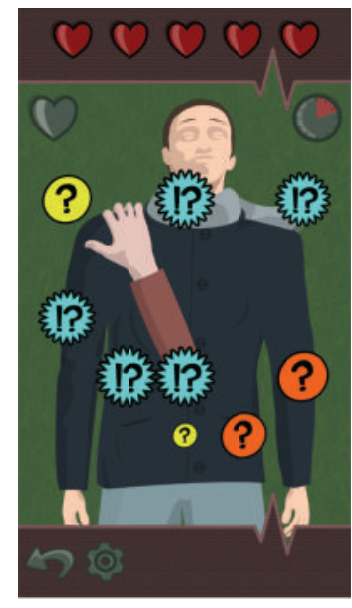

(c)

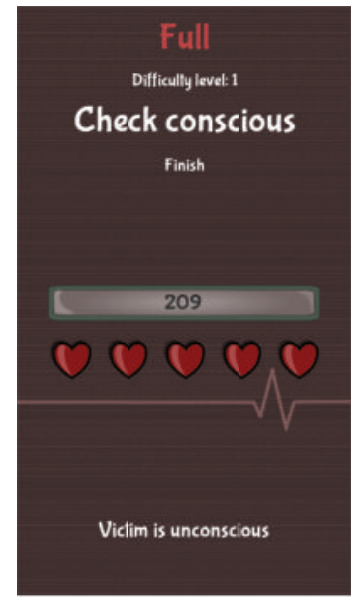

(d)

FIGURE 5: Screens of the Check Conscious mini game. (a) Init screen with game instructions. (b) and (c) scenes with the icons that have to be pressed to shake shoulder, only blue and yellow icons add points. (d) Final screen with the game score and the message that the victim is unconscious.

playing the next mini game, Scores to check the score, and Exit to exit the game); the menu to select the mini game we want to play (see Figure 3(c)); and the selector of the difficulty level once the game has been selected (see Figure 3(d)).

Figure 4 corresponds to the Isolate Victim mini game. From (a) to (d), we can see the init screen with game instructions and a timer that indicates when the game will start. We can also see the number of lives that the player has, in this case five hearts (see Figure 4(a)). This screen configuration is the same for all the mini games. The second image indicates what we have to do to isolate the victim. Note that we have hearts of different colours (see Figure 4(b)). Once we have pressed on the broken heart, the security perimeter appears and we have to isolate the victim as the message indicates (see Figure 4(c)). The last screen is the one that appears when the game is over. It indicates that the victim and the helper are in a secure position (see Figure 4(d)).

Figure 5 corresponds to the Check Conscious mini game. From (a) to (d), we can see the init screen with game instructions (see Figure 5(a)). The second image indicates what we have to do to check consciousness: press blue and yellow icons (see Figure 5(b)). Once we have pressed on the correct icons we will see how the hand moves the victim's shoulder (see Figure 5(c)). The last screen indicates that victim is unconscious (see Figure 5(d)).

Figure 6 corresponds to the Ask for Help mini game. From (a) to (d), we can see the init screen with game instructions (see Figure 6(a)). The second image indicates what we have to do to ask for help and also the icons with letters falling in the screen (see Figure 6(b)). Once correct 


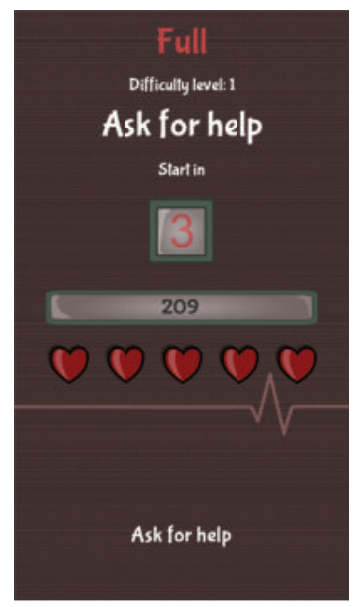

(a)

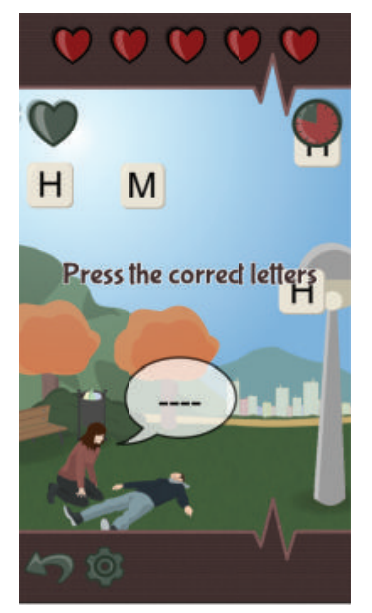

(b)

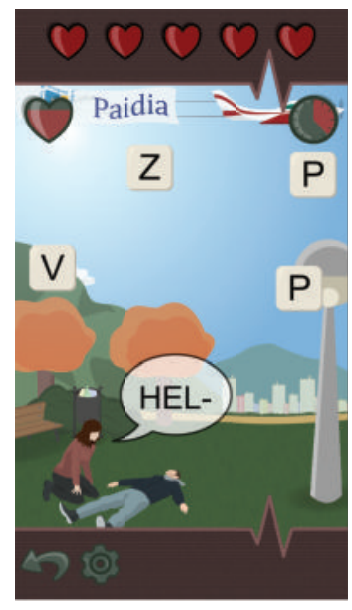

(c)

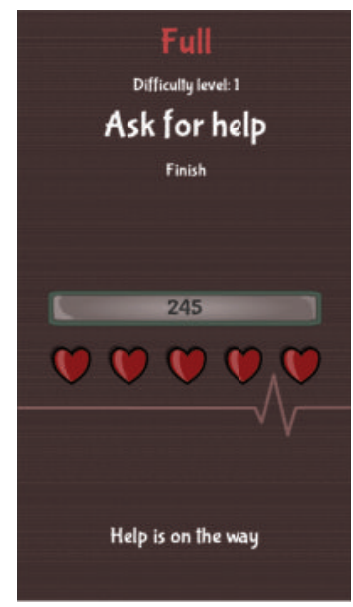

(d)

FIGURE 6: Screens of the Ask for Help mini game. (a) Init screen with game instructions. (b) Scene with the empty balloon and falling letters. (c) Scene with some of the help letters completed. (d) Final screen with the game score and the message that help is on the way.

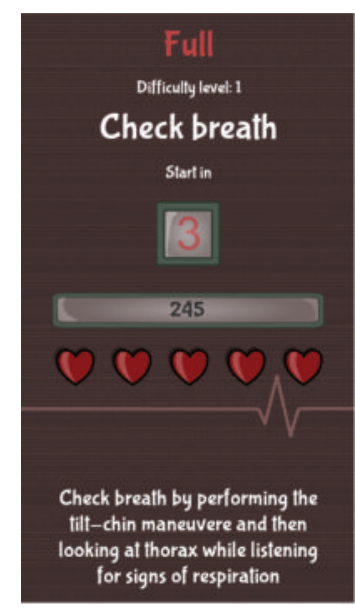

(a)

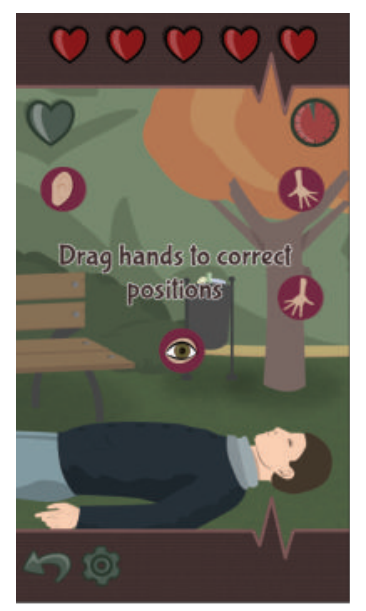

(b)

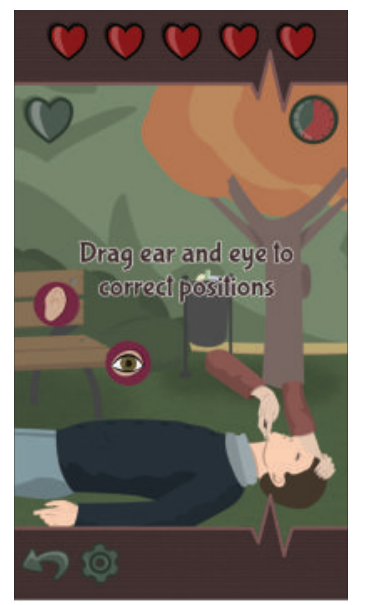

(c)

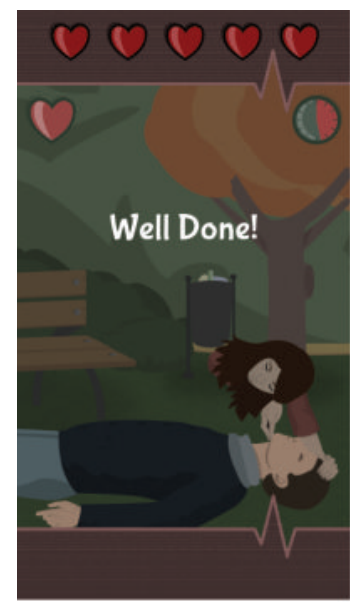

(d)

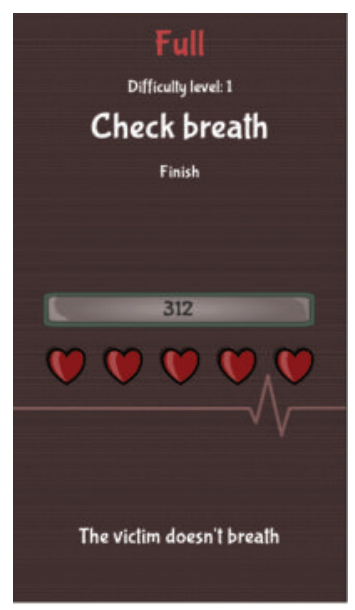

(e)

FiguRE 7: Screens of the Check Breath mini game. (a) Init screen with game instructions. (b) Scene with the icons that have to be placed in the correct positions. (c) Tilt-chin maneuver once hands have been correctly placed. (d) Look and listen for breath once eye and ear icons have been correctly placed. (e) Final screen with the score and the message that the victim is not breathing. 


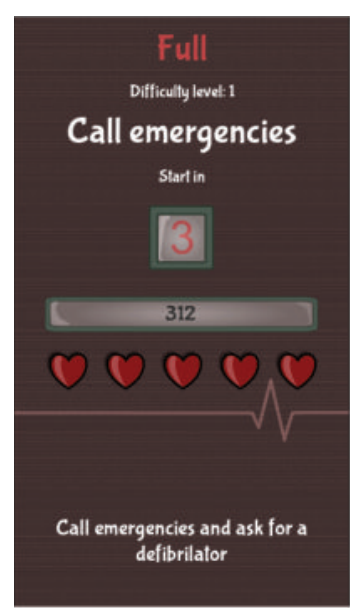

(a)

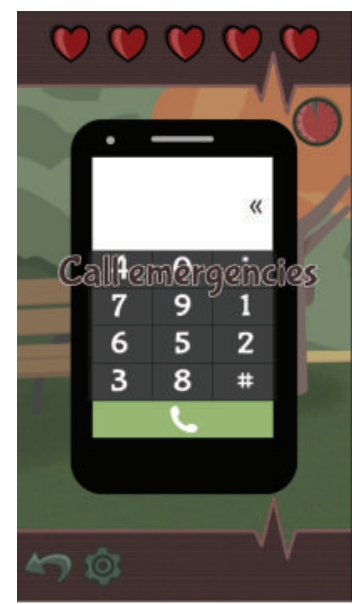

(b)

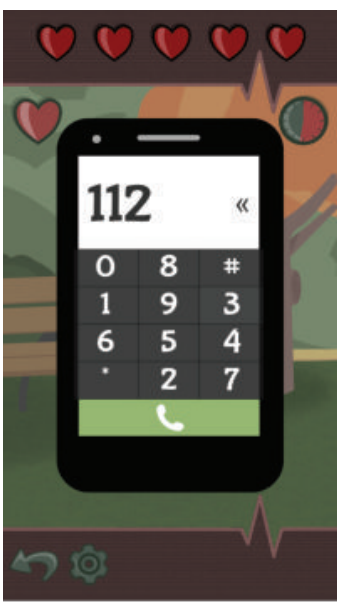

(c)

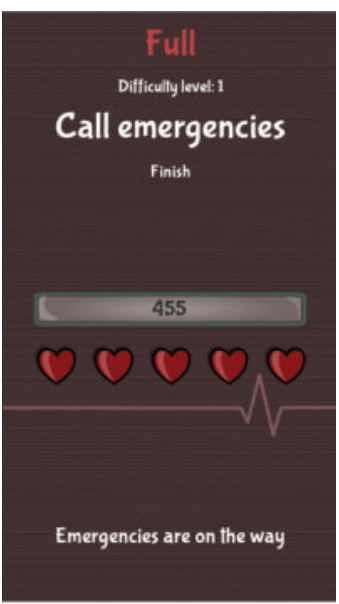

(d)

FIGURE 8: Game screens corresponding to the Ask for Help. (a) Init screen with game instructions. (b) Main screen with phone numbers and (c) once the player has dialed the correct number. (d) Final screen with the obtained score and the message that help and defibrillator are on the way.

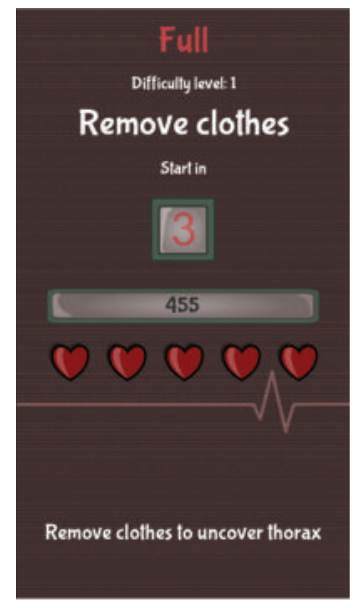

(a)

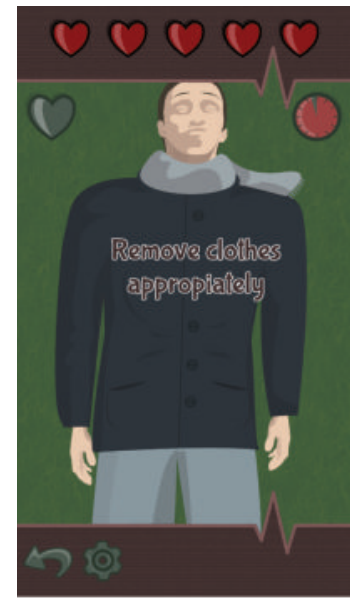

(b)

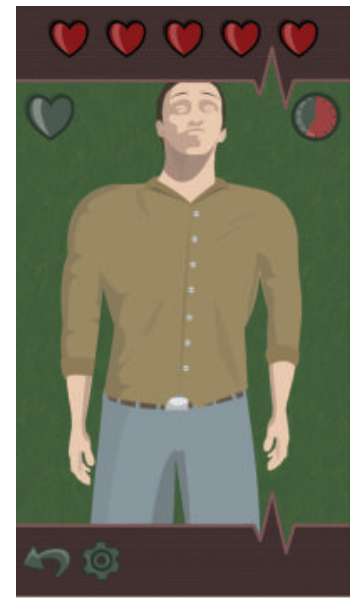

(c)

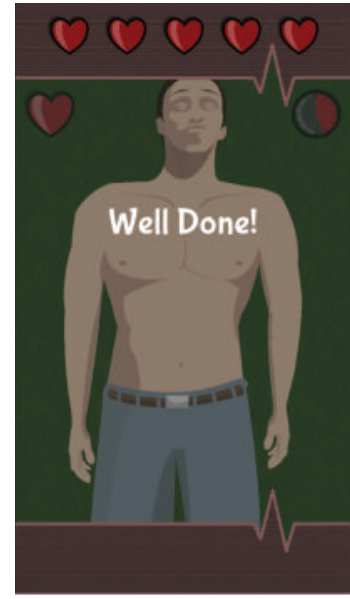

(d)

FIGURE 9: Game screens corresponding to the Remove Clothes mini game. (a) Init screen with game instructions. (b) Main scene with the victim wearing all the clothes. (c) After removing the scarf and the jacket. (d) Victim with the uncovered thorax.

letters are selected they appear in the speech balloon (see Figure 6(c)). The last screen indicates that help is on the way (see Figure 6(d)).

Figure 7 corresponds to the Check Breath mini game. From (a) to (e), we can see the init screen with game instructions indicating that we have to perform the tilt-chin maneuver and look and listen for breath (see Figure 7(a)). The second image shows the different icons of the game. As you can see, there are left and right hands to perform the tilt-chin maneuver and eyes and ears to look and listen. The icons have to be placed in the correct parts of the body victim (see Figure 7(b)). Once hands are correctly placed, the helper hands appear to show the correct hands placement on the victim (see Figure $7(\mathrm{c})$ ). The next screen shows the helper head position which appears once eye and ear icons are correctly placed (see Figure 7(d)). The last screen indicates that the victim is not breathing (see Figure $7(\mathrm{e})$ ).

Figure 8 corresponds to the Call Emergencies mini game. From (a) to (d), we can see the init screen with game instructions (see Figure 8(a)). The second image shows the phone with all the numbers (see Figure 8(b)). During the game, the numbers change their positions (see Figure 8(c)). The last screen indicates that emergencies are on the way (see Figure 8(d)).

Figure 9 corresponds to the Remove Clothes mini game. From (a) to (d), we can see the init screen with game instructions that indicate we have to uncover the victim thorax (see Figure 9(a)). The second image shows the victim with all the clothes (see Figure 9(b)). The next one shows the victim once the scarf and the jacket have been removed 


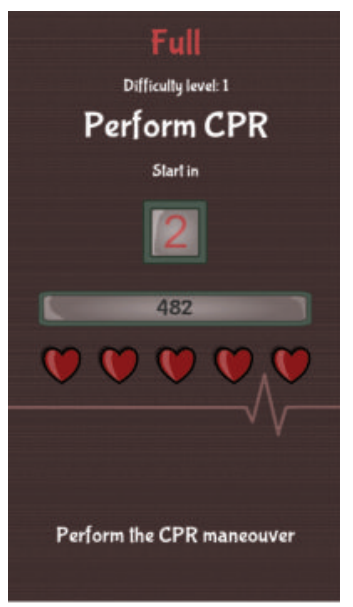

(a)

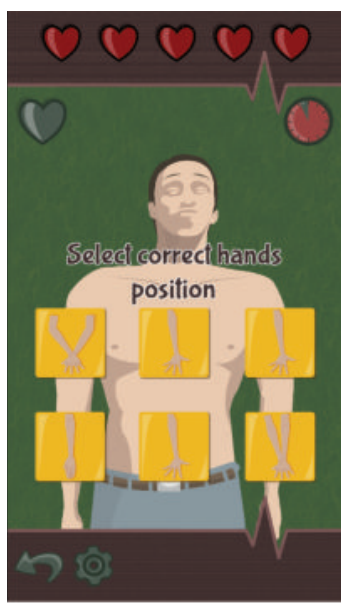

(b)

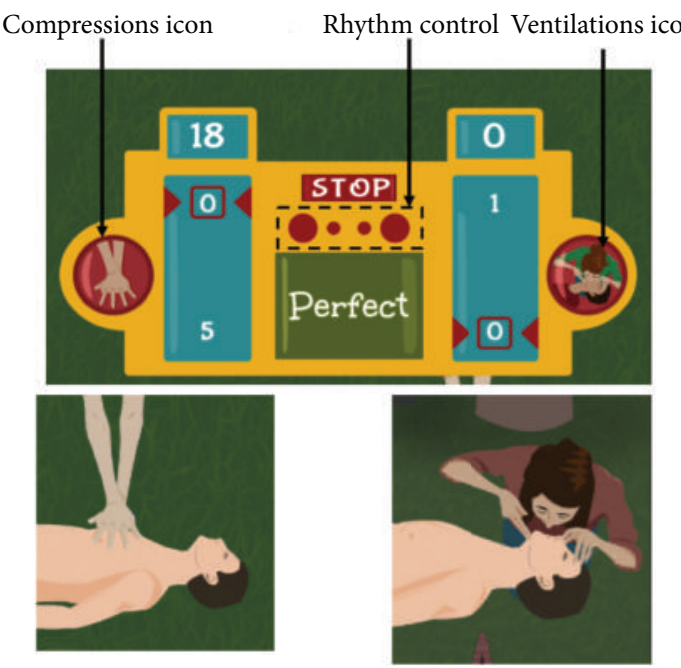

(c)

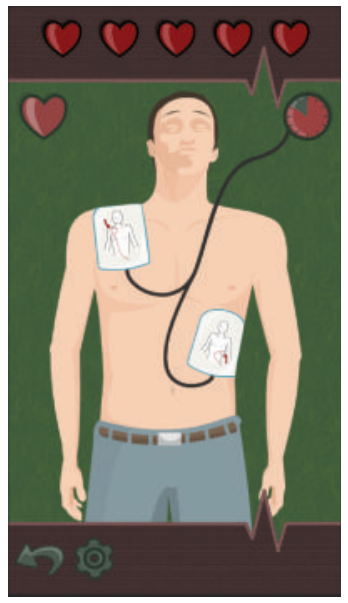

(d)

Figure 10: Game screens corresponding to the Perform CPR mini game. (a) The init screen. (b) The icon to select correct hand positions. (c) The menu to perform compressions and ventilations. (d) Defibrillator patches after performing CPR maneuver.

(see Figure 9(c)). The last one shows the victim with the uncovered thorax (see Figure 9(d)).

Figure 10 corresponds to the Perform CPR mini game which represents the key point of the protocol. From (a) to (d), we can see the init screen with game instructions (see Figure 10(a)). To start the game, the player has to select the correct hand positions. There will appear icons with different hands positions and the player has to select the correct one (see Figure 10(b)). Figure 10(c) represents the menu designed to control compressions and ventilations performance. In the middle of this board we can see if the performed action has been correct or not. Note that in the board we can also see the number of compressions and ventilations that have been performed and also indicators of the compression depth and ventilation degree. Once the maneuver has finished the defibrillator arrives and the player has to place the patches in the correct place (see Figure 10(d)).
Figure 11 corresponds to the Security Position mini game. From (a) to (e), we can see the init screen with game instructions (see Figure 11(a)). To start the game, the player has to move the victim by pressing on arms or legs of his body (see Figure 11(b)). Once the correct part is pressed, an icon appears as the one showed at the bottom of Figure 11(c). In next figure, we can see the victim after moving his arm (see Figure 11(d)). The last image represents the victim in the lateral security position (see Figure 11(e)).

4.1. First Users' Impressions. To test the game we selected 25 children, five of each age from 8 to 12 years old, and 12 males and 13 females. They played the game as many times as they wanted and then, we asked for clarity of instructions and three settings of the game: the aesthetics of scenarios, the playability, and the enjoyability of each mini game. For all four questions users should answer their impression in a scale 


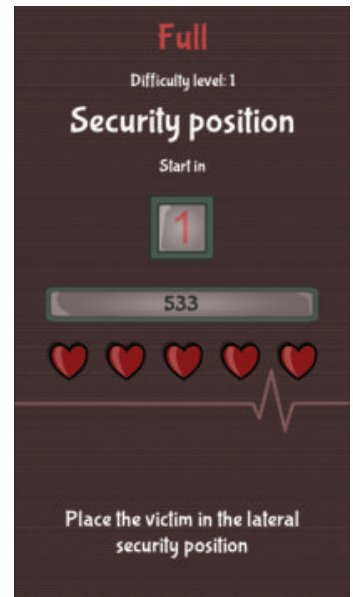

(a)

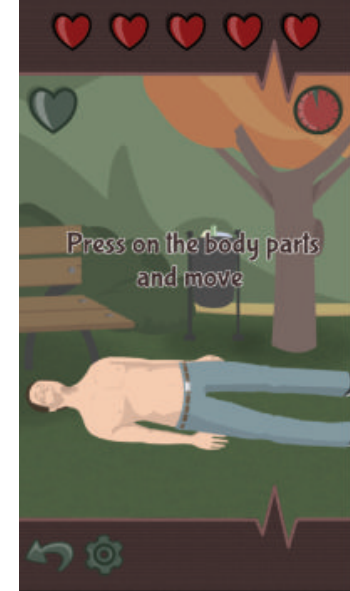

(b)

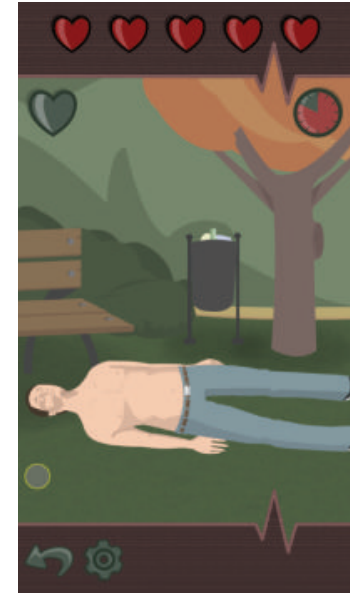

(c)

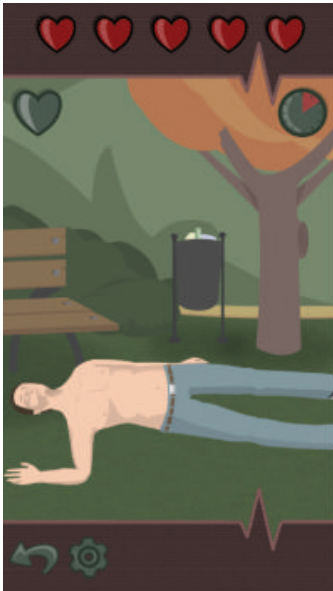

(d)

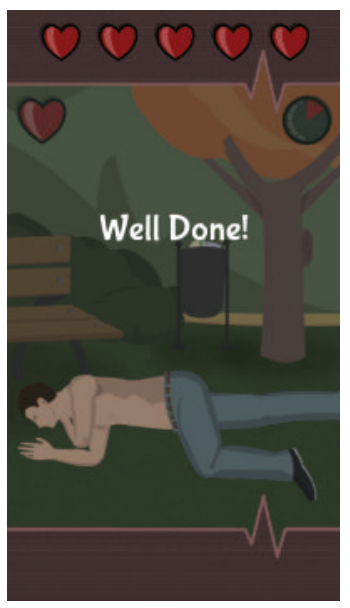

(e)

FIGURE 11: Game screens corresponding to the Security Position mini game. (a) The main scene. (b) The icon that appears to move the arm. (c) After moving the arm. (d) The victim in the lateral security position.

from 1 to 5 where 1 recalls to I do not like at all and 5 to I like a lot. In order to summarize the results, a mean of all three settings was calculated. Table 1 shows mean and standard deviation of answers. Regarding the clarity of instructions, all 8 mini games have values higher than 3 and 6 of them $(1,2,3$, 5,6 , and 7 ) even higher than 4 . On the other hand, the settings mean presents the same results except in Game 8 with a mean of only 2,43. The last column of Table 2 shows there is no significant difference between sexes on the answer, according the $p$ values obtained by a Mann-Whitney $U$ test. Due to the low size of our sample we plan to repeat the experiment with a larger one.

\section{Conclusions and Future Work}

The correct application of cardiopulmonary resuscitation protocol can increase the chances of survival for victims of cardiac arrest. Due to the importance of this procedure, many initiatives have been proposed to disseminate it. However, it is still unknown for a large number of people, and new strategies to promote it are needed. In this paper, we have proposed a game with this purpose and specially targeted to children. Following the cardiopulmonary resuscitation guidelines proposed by the European Resuscitation Council 2010 and assessed by experts, we have converted the main procedures of these guidelines into eight different mini games. Each mini game represents one of the key processes of the protocol and is presented to the player as a challenge. Playing with them makes the player learn the protocol. Our first experiments show us the suitability of the game as a channel to disseminate the protocol, mainly among children. As an immediate work we will update the game to adapt it to the ERC guidelines proposed in 2015 and we will create/improve the scenarios and challenges to preserve the player interest. For instance, in mini game five, we will include the dispatcher assisted CPR instruction. Moreover, we will carry out a further evaluation with a larger sample. In addition, we will evaluate the efficacy of the game to improve the willingness and quality of basic life support in a clinical setting. 
TABLE 2: Mean and standard deviation of 25 testers' answers related to clarity of instructions and three settings of the game: the aesthetics of scenarios, the playability, and the enjoyability of each mini game.

\begin{tabular}{lcccc}
\hline & & Mean & Standard deviation & Mann-Whitney $U$ \\
& & 4,28 & 0,61 & 1,00 \\
Game 1 & Clarity & 4,16 & 0,43 & 0,17 \\
Isolate Victim & Settings mean & 4,36 & 0,57 & 0,53 \\
\hline Game 2 & Clarity & 4,12 & 0,41 & 0,96 \\
Check Conscious & Settings mean & 4,56 & 0,51 & 0,08 \\
\hline Game 3 & Clarity & 4,24 & 0,35 & 0,59 \\
Ask for Help & Settings mean & Clarity & 3,68 & 0,75 \\
\hline Game 4 & Settings mean & 3,52 & 0,42 & 0,40 \\
Check Breath & Clarity & 4,56 & 0,51 & 0,85 \\
\hline Game 5 & Settings mean & 4,45 & 0,29 & 0,61 \\
Call Emergencies & Clarity & 4,46 & 0,51 & 0,85 \\
Game 6 & Settings mean & 4,19 & 0,36 & 0,41 \\
Remove Clothes & Clarity & 4,16 & 0,55 & 0,51 \\
\hline Game 7 & Settings mean & 4,16 & 0,59 & 0,27 \\
Perform CPR & Clarity & 3,4 & 0,58 & 1,00 \\
\hline Game 8 & Settings mean & 2,43 & 0,46 & 0,91 \\
Security Position & & & \\
\hline
\end{tabular}

\section{Competing Interests}

There are no competing interests.

\section{Acknowledgments}

This work has been funded in part by grants from the Spanish Government (no. TIN2013-47276-C6-1-R) and from the Catalan Government (no. 2014-SGR-1232).

\section{References}

[1] M. Prensky, Digital Game-Based Learning, McGraw Hill, New York, NY, USA, 2001.

[2] T. M. Connolly, E. A. Boyle, E. MacArthur, T. Hainey, and J. M. Boyle, "A systematic literature review of empirical evidence on computer games and serious games," Computers and Education, vol. 59, no. 2, pp. 661-686, 2012.

[3] J. P. Gee, "Learning by design: good video games as learning machines," E-Learning, vol. 2, no. 1, pp. 5-16, 2005.

[4] D. W. Shaffer, How Computer Games Help Children Learn, Palgrave Macmillan, New York, NY, USA, 2006.

[5] F. Ke, "A case study of computer gaming for math: engaged learning from gameplay?" Computers and Education, vol. 51, no. 4, pp. 1609-1620, 2008.

[6] M. Kebritchi and A. Hirumi, "Examining the pedagogical foundations of modern educational computer games," Computers and Education, vol. 51, no. 4, pp. 1729-1743, 2008.

[7] H. F. O'Neil, R. Wainess, and E. L. Baker, "Classification of learning outcomes: evidence from the computer games literature," Curriculum Journal, vol. 16, no. 4, pp. 455-474, 2005.

[8] M. Virvou and E. Alepis, "Mobile educational features in authoring tools for personalised tutoring," Computers and Education, vol. 44, no. 1, pp. 53-68, 2005.
[9] P. Lavín-Mera, P. Moreno-Ger, and B. Fernández-Manjón, "Development of educational videogames in m-learning contexts," in Proceedings of the 2nd IEEE International Conference on Digital Game and Intelligent Toy Enhanced Learning (DIGITEL '08), pp. 44-51, Banff, Canada, November 2008.

[10] T. Kitamura, T. Iwami, T. Kawamura et al., "Nationwide improvements in survival from out-of-hospital cardiac arrest in Japan," Circulation, vol. 126, no. 24, pp. 2834-2843, 2012.

[11] T. Nishi, T. Maeda, K. Takase, T. Kamikura, Y. Tanaka, and H. Inaba, "Does the number of rescuers affect the survival rate from out-of-hospital cardiac arrests? Two or more rescuers are not always better than one," Resuscitation, vol. 84, no. 2, pp. 154161, 2013.

[12] Y. Tanaka, J. Taniguchi, Y. Wato, Y. Yoshida, and H. Inaba, "The continuous quality improvement project for telephone-assisted instruction of cardiopulmonary resuscitation increased the incidence of bystander CPR and improved the outcomes of outof-hospital cardiac arrests," Resuscitation, vol. 83, no. 10, pp. 1235-1241, 2012.

[13] J. Herlitz, J. Engdahl, L. Svensson, M. Young, K.-A. Ängquist, and S. Holmberg, "Characteristics and outcome among children suffering from out of hospital cardiac arrest in Sweden," Resuscitation, vol. 64, no. 1, pp. 37-40, 2005.

[14] G. Abbas, A. Alfonzo, H. R. Arntz et al., "European Resuscitation Council Guidelines for Resuscitation 2010 Section 1. Executive summary," Resuscitation, vol. 81, no. 10, pp. 1219-1276, 2010.

[15] W. B. Kouwenhoven, J. R. Jude, and G. Knickerbocker, "Closedchest cardiac massage," Journal of the American Medical Assocition, vol. 173, no. 10, pp. 1064-1067, 1960.

[16] American Heart Association, "Emergency Cardiovascular Care online education," http://www.onlineaha.org.

[17] Y. T. Fong, V. Anantharaman, S. H. Lim, K. F. Leong, and G. Pokkan, "Mass cardiopulmonary resuscitation 99-Survey results of a multi-organisational effort in public education in 
cardiopulmonary resuscitation," Resuscitation, vol. 49, no. 2, pp. 201-205, 2001.

[18] E. L. Einspruch, B. Lynch, T. P. Aufderheide, G. Nichol, and L. Becker, "Retention of CPR skills learned in a traditional AHA Heartsaver course versus 30-min video self-training: a controlled randomized study," Resuscitation, vol. 74, no. 3, pp. 476-486, 2007.

[19] C. Vaillancourt, J. Grimshaw, J. C. Brehaut et al., "A survey of attitudes and factors associated with successful cardiopulmonary resuscitation (CPR) knowledge transfer in an older population most likely to witness cardiac arrest: design and methodology," BMC Emergency Medicine, vol. 8, article 13, 2008.

[20] A.-B. Thoren, A. Axelsson, and J. Herlitz, "The attitude of cardiac care patients towards CPR and CPR education," Resuscitation, vol. 61, no. 2, pp. 163-171, 2004.

[21] Medtronic Foundation, CPR Simulator, 2012, http://www.cprsim.com/.

[22] CPR Choking, 2011, http://www.stonemeadowdevelopment.com/ StoneMeadowDevelopment/CPR\&Choking.html.

[23] EM Gladiators LLC, CPR Game, 2011, http://www.freenew.net/ iphone/cpr-game-20/466678.htm.

[24] D. Low, N. Clark, J. Soar et al., "Does use of iResus $\odot$ application on a smart phone improve the performance of an advanced life support provider in a simulated emergency?" Resuscitation, vol. 81, no. 2, supplement, p. S10, 2010.

[25] A. Alessandri, iCPR-iPhone App for CPR Training, 2009, http:// www.icpr.it/.

[26] F. Semeraro, F. Taggi, G. Tammaro, G. Imbriaco, L. Marchetti, and E. L. Cerchiari, "ICPR: a new application of high-quality cardiopulmonary resuscitation training," Resuscitation, vol. 82, no. 4, pp. 436-441, 2011.

[27] R. Zanner, D. Wilhelm, H. Feussner, and G. Schneider, "Evaluation of M-AID, a first aid application for mobile phones," Resuscitation, vol. 74, no. 3, pp. 487-494, 2007.

[28] LifeSaver, 2012, https://life-saver.org.uk/.

[29] Indiegogo Inc. Mini-VREM, 2012, http://www.mini-vrem.it/.

[30] Insight Instructional Media, AED Challenge, 2011, http://aedchallenge.com/.

[31] M. Ponder, B. Herbelin, T. Molet et al., "Interactive scenario immersion: health emergency decision training in just project," in Proceedings of the International Workshop on Virtual Reality Rehabilitation, pp. 87-101, 2002.

[32] Laerdal Medical, "MicroSim Prehospital," 2012, http://www .laerdal.com/gb/docid/12984879/MicroSimPrehospital.

[33] Ilumens, Staying Alive 3D, 2011, http://www.stayingalive.fr/ index_uk.html.

[34] V. Wattanasoontorn, I. Boada, and M. Sbert Lissa, "A serious game to learn cardiopulmonary resuscitation," in Proceedings of the 8th International Conference on Foundations of Digital Games, Games for Learning Workshop (FDG '13), 2013.

[35] F. Semeraro, A. Frisoli, G. Ristagno et al., "Relive: a serious game to learn how to save lives," Resuscitation, vol. 85, no. 7, pp. e109e110, 2014.

[36] Viva! Game, http://www.viva2013.it/viva-game.

[37] B. Schmitz, R. Klemke, J. Walhout, and M. Specht, "Attuning a mobile simulation game for school children using a designbased research approach," Computers and Education, vol. 81, pp. 35-48, 2015.

[38] T. Uray, A. Lunzer, A. Ochsenhofer et al., "Feasibility of Lifesupporting First-aid (LSFA) training as a mandatory subject in primary schools," Resuscitation, vol. 59, no. 2, pp. 211-220, 2003.
[39] M. S. Link, “CPR for kids: please try this at home," Journal Watch Cardiology, vol. 28, pp. 234-239, 2007.

[40] I. Jones, R. Whitfield, M. Colquhoun, D. Chamberlain, N. Vetter, and R. Newcombe, "At what age can schoolchildren provide effective chest compressions? An observational study from the Heartstart UK schools training programme," British Medical Journal, vol. 334, no. 7605, pp. 1201-1203, 2007.

[41] D. L. Isbye, L. S. Rasmussen, C. Ringsted, and F. K. Lippert, "Disseminating cardiopulmonary resuscitation training by distributing 35000 personal manikins among school children," Circulation, vol. 116, no. 12, pp. 1380-1385, 2007.

[42] American Academy of Pediatrics Committee on Injury, Violence, and Poison Prevention, "Prevention of drowning in infants, children, and adolescents," Pediaitrics, vol. 112, pp. 437439, 2003.

[43] L. A. Pyles and J. Knapp, "Role of pediatricians in advocating life support training courses for parents and the public," Pediatrics, vol. 114, no. 6, pp. e761-e765, 2004.

[44] J. Torrente, B. Borro-Escribano, M. Freire et al., "Development of game-like simulations for procedural knowledge in healthcare education," IEEE Transactions on Learning Technologies, vol. 7, no. 1, pp. 69-82, 2014.

[45] J. Dewey, Experience and Education, Simon and Schuster, New York, NY, USA, 1938.

[46] R. C. Schank, T. R. Berman, and K. A. Macpherson, "Instructional-design theories and models: a new paradigm of instructional theory," in Learning by Doing, C. M. Reigeluth and N. J. Mahwah, Eds., pp. 633-651, Lawrence Erlbaum Associates, Mahwah, NJ, USA, 1999.

[47] E. L. Deci and R. M. Ryan, Intrinsic Motivation and SelfDetermination in Human Behavior, Plenum, New York, NY, USA, 1985.

[48] E. L. Deci and R. M. Ryan, "The "what" and "why" of goal pursuits: human needs and the self-determination of behavior," Psychological Inquiry, vol. 11, no. 4, pp. 227-268, 2000.

[49] C. Burgers, A. Eden, M. D. van Engelenburg, and S. Buningh, "How feedback boosts motivation and play in a brain-training game," Computers in Human Behavior, vol. 48, pp. 94-103, 2015. 


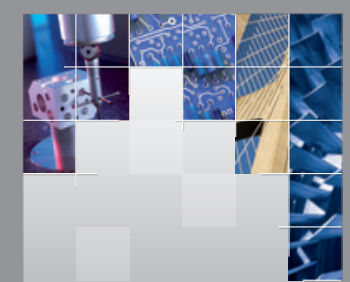

\section{Enfincering}
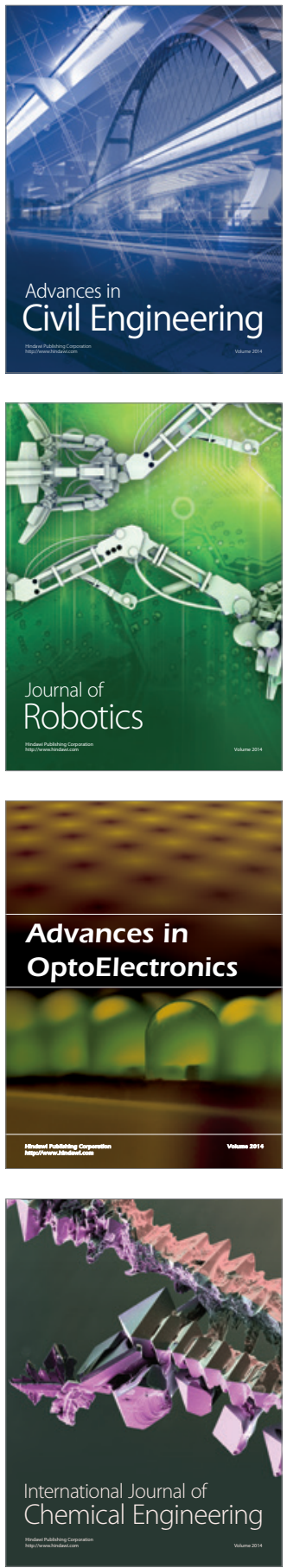

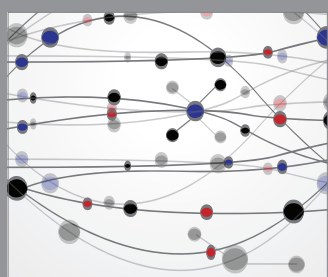

The Scientific World Journal

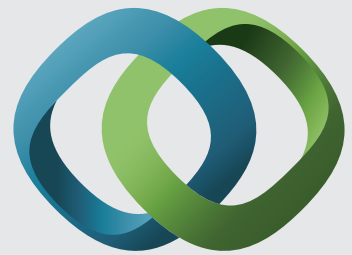

\section{Hindawi}

Submit your manuscripts at

http://www.hindawi.com
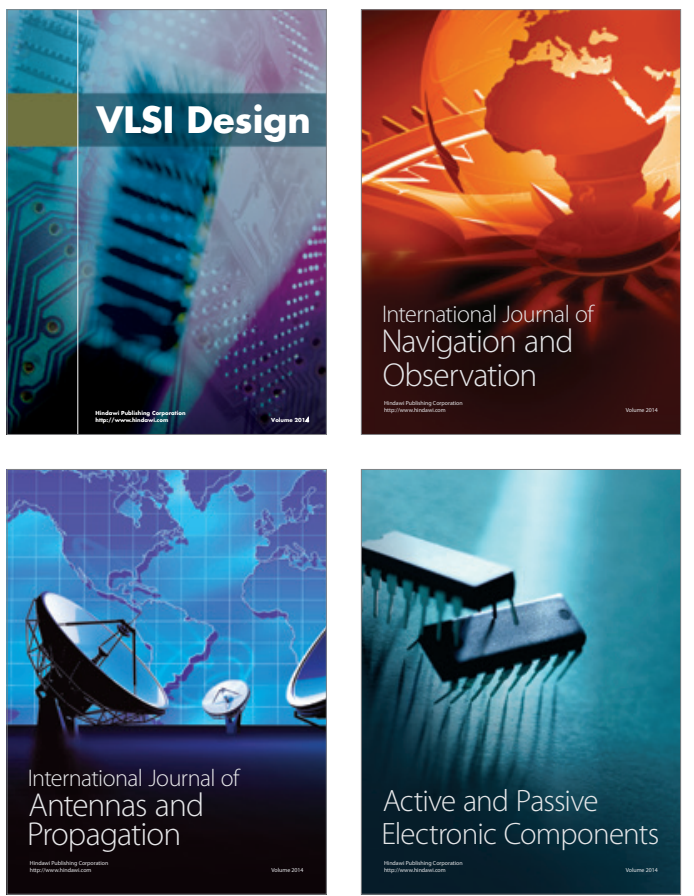
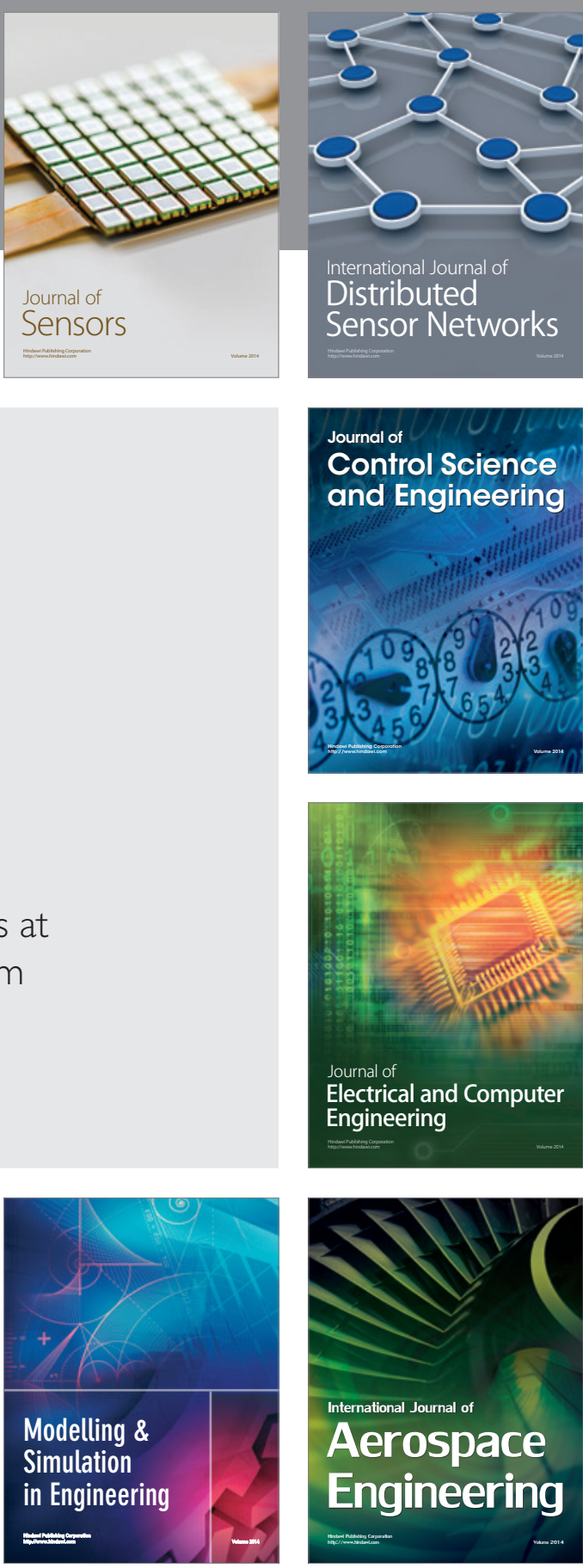

International Journal of

Distributed

Sensor Networks

Journal of

Control Science

and Engineering
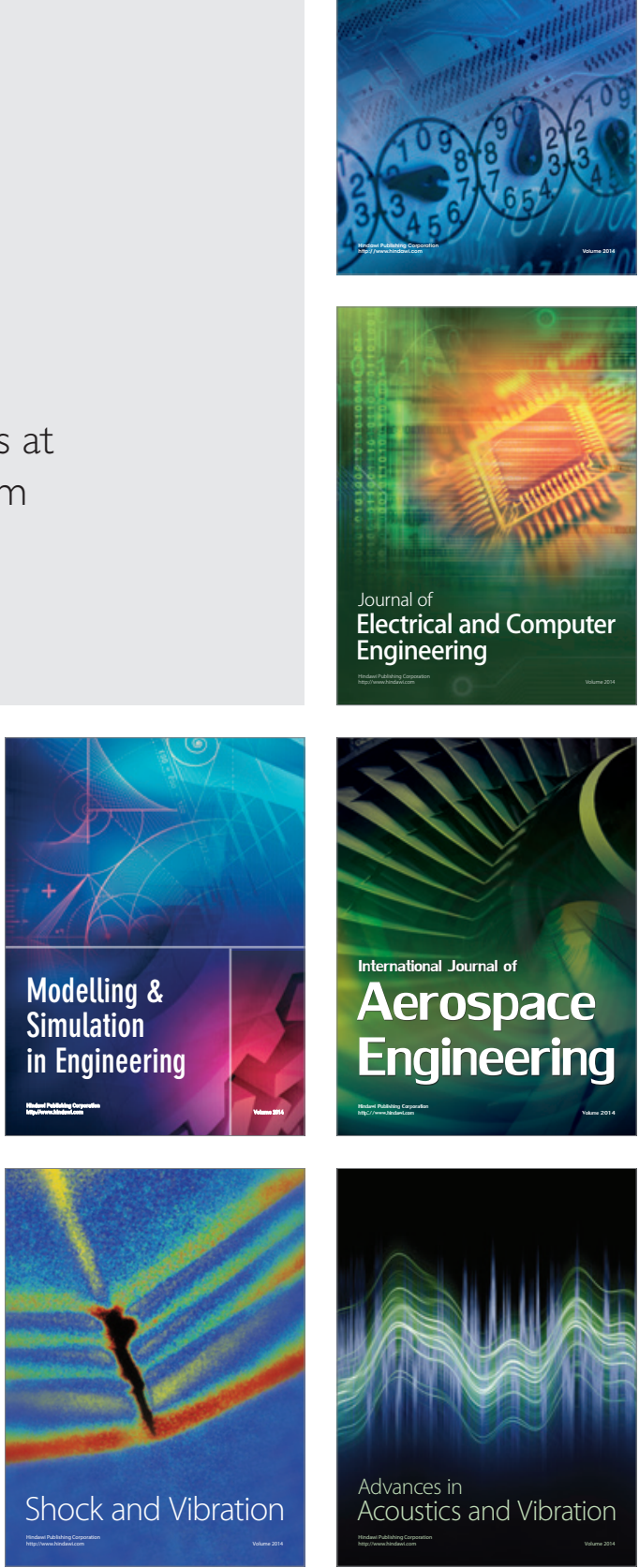